\title{
More QTL for flowering time revealed by substitution lines in Brassica oleracea
}

\author{
A. M. RAE, E. C. HOWELL \& M. J. KEARSEY* \\ Plant Genetics and Cell Biology Group, School of Biological Sciences, The University of Birmingham, Birmingham \\ B15 2TT, U.K.
}

\begin{abstract}
Seventy-nine recombinant backcross substitution lines from a cross between Brassica oleracea var. italica and Brassica oleracea var. alboglabra were grown in field trials over five years along with the alboglabra recurrent parent. Plants were scored for the days from sowing to the opening of the first flower, and lines that flowered significantly earlier or later than the recurrent parent were identified. Based on the lengths of the substitutions, evidence for 11 QTL on chromosomes O1, O2, O3, O5 and O9 was found, five of which mapped to similar regions to five of the six found in a previous analysis of doubled haploid lines from the same cross. Several of the QTL were linked closely in repulsion.
\end{abstract}

Keywords: Brassica, flowering time, QTL analysis, substitution lines.

\section{Introduction}

Brassica oleracea is an agriculturally important species including many vegetables such as cabbage, broccoli and cauliflower. It is closely related to the model dicotyledonous plant Arabidopsis thaliana and so it is expected that information concerning control of basic biological processes in A. thaliana will be transferable to Brassica crops.

Information should also be transferable between different species within the Brassica genus, as the three diploid genomes, A, B and C, of B. rapa, B. nigra and $B$. oleracea, respectively, are thought to have derived from the same ancestor. These genomes reveal striking conservation of content, although chromosome duplication and translocation have occurred during divergence (Lagercrantz \& Lydiate, 1996). The amphidiploid B. napus (oilseed rape) genome is made up of the $\mathrm{A}$ and $\mathrm{C}$ genomes probably from close relatives of B. rapa and B. oleracea. (U, 1935; Parkin et al., 1995).

Flowering time is not only of scientific interest because it facilitates the understanding of plant development, it is also important in agriculture, because its modification may enable the geographical range of the Brassica crop to be extended. For example, cultivation of B. napus is usually restricted to temperate latitudes, but the development of early flowering cultivars would allow the crop to be grown in low-rainfall regions such as the Western Australian wheat belts (Thurling \&

*Correspondence. E-mail: m.j.kearsey@bham.ac.uk
Depittayanan, 1992), and more northern regions of Canada (Murphy \& Scarth, 1994).

Genes affecting flowering time have been identified in A. thaliana by mutagenesis (Koornneef et al., 1991). For example, mutations of the CONSTANS gene cause delayed flowering under long days, but not short, and the gene has been cloned by chromosome walking (Putterill et al., 1993, 1995).

Two regions influencing flowering time in B. nigra (on LG2 and LG8) have been found to be homologous to the CONSTANS gene region (Lagercrantz et al., 1996). These regions have also been shown to carry quantitative trait loci (QTL) in the A genome of B. napus, and show large-scale colinearity between regions on chromosomes $\mathrm{O} 2, \mathrm{O} 3$ and $\mathrm{O} 9$ of B. oleracea.

Previous studies mapping QTL for flowering time in B. oleracea have been reported. For example, Kennard et al. (1994) found significant QTL using single-factor ANOVA on an $F_{2}$ from a cabbage $\times$ broccoli cross, whereas Camargo \& Osborn (1996) used $F_{3}$ families from a different cross between cabbage and broccoli. Because of the lack of both a standardized nomenclature for linkage groups in Brassica species and of common probes, it is not possible to compare the QTL locations found in these studies. Another drawback to these studies is the limited life-span of the populations used. The heterozygosity of individuals prevents their maintenance by selfing, so trials can only be carried out once in a single environment. Bohuon et al. (1998) overcame this by using doubled haploid lines derived from the $F_{1}$ of a cross between a Chinese kale and a hybrid of 
calabrese, resulting in six QTL for flowering time being mapped to chromosomes $\mathrm{O} 2, \mathrm{O} 3, \mathrm{O} 5$ and $\mathrm{O} 9$.

The use of doubled haploid plants still has the limitation that segregating populations can map QTL only to a relatively large region of a chromosome. This present paper reports work which reinforces the results of Bohuon et al. (1998) by using the same cross to engineer near-isogenic lines. Repeated backcrossing and selfing of the progeny from this cross have enabled the production of lines that contain small segments of donor parent DNA within a recurrent parent background (Ramsay et al., 1996). Differences in phenotype between the lines and the recurrent parent should be caused by the substituted region. The substitution lines should be homozygous so that the lines can be maintained and multiplied by selfing allowing the same lines to be grown in different environments so that experiments can be repeated over a number of years and conditions. The precision to which a QTL can be mapped depends upon the size of the introgressed region, but comparisons between different substitution lines can narrow these regions further.

\section{Materials and methods}

\section{Production of experimental lines}

The parent plants were descended from microsporederived doubled haploid lines. The recurrent parent is A12DHd, derived from Brassica oleracea var. alboglabra, and the donor is GDDH33, which is Brassica oleracea var. italica, derived from a commercial calabrese $\mathrm{F}_{1}$ hybrid variety, Green Duke (Bohuon et al., 1996). For simplicity, the doubled haploid lines used as parents will be referred to as A12 and GD, respectively.

The substitution lines were produced (as described in Ramsay et al., 1996) by using a single $\mathrm{F}_{1}(\mathrm{~A} 12 \times \mathrm{GD})$ to pollinate three $\mathrm{A} 12$ plants to produce the first backcross generation $\left(\mathrm{BC}_{1}\right) . \mathrm{A} 12$ was used as the female to ensure that all cytoplasm from the first backcross generation and subsequent generations was A12 in origin. Lines were selected for a high number of purely recurrent linkage groups, wide coverage of the genome by the donor introgression and duplications of introgressed regions. Lines were backcrossed again and then selfed two or three times to produce lines with homozygous regions of introgressed GD DNA in an A12 background.

Seventy-eight RFLP probes selected from those previously described by Bohuon et al. (1996) to construct a linkage map from doubled haploid lines produced from the $F_{1}$ of this cross, were used to map the substituted regions in the lines together with an additional 119 AFLP markers (Sebastian et al.
1999). This gave an average spacing between markers of $4.5 \mathrm{cM}$. The positions of the introgressed GD DNA in the 79 substitution lines are shown in Appendix 1. The minimum substitution indicates the region known to be GD DNA, whereas the maximum substitution includes the region between A12 and GD markers in which recombination has occurred. It is not yet possible to define the proportion of this region which is GD, so the whole section must be taken into account.

\section{Trial plan}

Eight field trials were carried out over a period of five years; one each in 1994 and 1995, two each in 1996, 1997 and 1998. Lines were used in trials as they became available from the breeding programme. Over the five years, 79 substitution lines, with varying numbers of replicates, were grown alongside the recurrent and the donor parents. Table 1 shows the sowing dates and the number of lines and replicates for each trial.

Seeds were sown in $5 \mathrm{~cm}$ plastic pots, using John Innes compost no. 3 , in the glasshouse and placed in a single block with complete individual plant randomization, surrounded by guard plants. This design was used to maximize the power to detect QTL (Kearsey \& Pooni, 1996). The glasshouse was unheated and unlit so that temperature and daylength depended on natural conditions.

After nine days the number of germinated seeds was noted, and plants were thinned from two to one per pot. Any pots in which no seed germinated were replaced with guard plants from the same line. The pots were moved to an open-ended polythene tunnel after 15 days for acclimatization. After 23 days the seedlings were planted in the field in the same random position. The field was set out in rows of 100 plants,

Table 1 The sowing dates for the eight Brassica oleracea trials over the five years, with the number of lines and the number of replicates. A12 and GD are the parent lines and SL denotes the substitution lines

\begin{tabular}{rccccc}
\hline Year & Trial Sowing date & A12 & GD & SL \\
\hline 1998 & T1 & $12 / 05 / 98$ & A12 $\times 200$ & GD $\times 25$ & $19 \mathrm{SL} \times 60$ \\
1998 & T2 & $26 / 05 / 98$ & A12 $\times 200$ & GD $\times 25$ & $19 \mathrm{SL} \times 60$ \\
1997 & T1 & $13 / 05 / 97$ & A12 $\times 200$ & GD $\times 30$ & $40 \mathrm{SL} \times 40$ \\
1997 & T2 & $27 / 05 / 97$ & A12 $\times 200$ & GD $\times 30$ & $40 \mathrm{SL} \times 40$ \\
1996 & T1 & $20 / 05 / 96$ & A12 $\times 250$ & - & $8 \mathrm{SL} \times 50$ \\
1996 & T2 & $12 / 06 / 96$ & A12 $\times 200$ & GD $\times 20$ & $23 \mathrm{SL} \times 50$ \\
1995 & T1 & $09 / 06 / 95$ & A12 $\times 200$ & GD $\times 20$ & $18 \mathrm{SL} \times 50$ \\
1994 & T1 & $01 / 06 / 94$ & A12 $\times 100$ & GD $\times 10$ & $21 \mathrm{SL} \times 20$ \\
\hline
\end{tabular}


spaced $76 \mathrm{~cm}$ between rows and $31 \mathrm{~cm}$ between plants in the rows. Guard plants were grown around the perimeter and in the spaces left by dead plants. The whole trial area was protected by nylon netting to prevent bird damage. Plants were irrigated during dry periods, and chemical treatments were used to control pests.

\section{Data collection}

Flowering was checked daily, and scored as the number of days from sowing to the first flower opening on each plant. Data from plants that were badly affected by cabbage root fly or grey aphid were removed. Other traits were also recorded, but their analysis will be published elsewhere.

\section{Data analysis}

1994, 1995 and 1996 trials Four one-way analyses of variance (ANOVAS) were carried out to test for variation between lines within each trial in 1994, 1995 and 1996. The within-lines mean squares from these ANovas were used to carry out Tukey-Kramer multiple comparison tests (Dunnett, 1980), to determine which substitution lines flowered significantly earlier or later than the A12 recurrent parent.

1997 trials Seeds for each substitution line in both trials in 1997 were taken from two different parent plants which had been grown in two completely randomized blocks in glasshouses. This was to allow maternal effects in the progeny to be estimated. The two field trials contained the same number of plants from the same parent plants so that variation between trials could also be examined.

The data for both trials were standardized to mean zero and variance one, to minimize genotype-byenvironment effects arising from different ranges in flowering time, so allowing the trials to be analysed together. A cross-classified hierarchical ANOvA was carried out for the substitution lines on the standardized data to test for interactions between genotypes and environments. This was repeated for the A12 parents in the two trials. The within-lines mean square for these ANOVAs were combined for use in a Tukey-Kramer multiple comparison test.

1998 trials The lines used in the 1998 field trials were selected as being earlier or later flowering than A12 based on the analysis of lines in previous years. This allowed the use of one-tailed tests to compare each line against the recurrent A12 parent. The data from each trial were standardized permitting the trials to be analysed together.

\section{Results}

\section{4, 1995 and 1996 trials}

The ANOvas for the four trials in 1994, 1995 and 1996 indicated significant variation between lines within trials, implying that the different substitutions of GD affected the flowering time of the lines.

The Tukey-Kramer test showed the lines SL112, SL113, SL140, SL175 in 1994; SL113, SL129, SL140, SL172 in 1995; SL102 in 1996 trial 1; and SL102, SL128, SL137, SL157, SL172, SL175 and SL178 in 1996 trial 2 were significantly later flowering (at the $5 \%$ level) than the A12 parent. Line SL108 was significantly earlier flowering than A12 in 1995.

\section{7 data}

The mean and standard deviation for flowering time for trial 1 and trial $2(\bar{x}=64.889, s=2.527$ and $\bar{x}=$ $65.476, s=4.041$, respectively) were used to standardize the data.

The cross-classified hierarchical ANOVA (Table 2a) showed that the variation between lines was highly significant $(P<0.001)$, and that replicate families within lines also differed $(0.01>P>0.05)$. The latter indicates the importance of maternal effects. The interaction of lines with trials, and the families within lines interaction with trials, were not significantly different from each other $(1.67 / 1.12=1.49, P>0.05)$, although families within lines did interact with trials significantly when compared to the error $(P<0.01)$. As expected, the variation between trials was very small $(P>0.05)$, any difference from zero being attributed to unequal numbers of observations in each trial.

A similar ANOVA was carried out for A12 recurrent parent plants (Table 2b), showing that the variation between different families within the A12 line and the variation between trials were not significant. However, interaction between trials and families was just significant despite the scalar transformation.

In order to carry out the Tukey-Kramer multiple comparison, the mean square 'between families within substitution lines' combined with the mean square 'between families' for the A12 was used as the error variation. The Tukey-Kramer test showed that lines SL115, SL134, SL141 and SL175 were significantly later flowering than the A12 recurrent parent.

\section{8 data}

Flowering data for the two trials in 1998 were standardized, using the mean and standard deviation for the two trials $(\bar{x}=68.417, s=3.742$ and $\bar{x}=64.257, s=3.551$, 
Table 2 (a). The cross-classified hierarchical ANOva for the Brassica oleracea substitution lines in 1997 trials 1 and 2 . The $F$-value is taken to be nonsignificant (NS) when $P>0.05$; *indicates $0.05>P>0.01$, * indicates $0.01>P>0.001$; ***indicates $P<0.001$ (b). The cross-classified ANOvA for the A12 recurrent parent in 1997 trials 1 and 2

(a)

\begin{tabular}{lcrrrrrr}
\hline Item & d.f. & \multicolumn{1}{c}{ Item } & d.f. & SS & MS & $F$ & $P$ \\
\hline Trial & 1 & Trial & 1 & 0.006 & 0.006 & 0.004 & NS \\
Families & 77 & Lines & 39 & 777.8 & 19.95 & 7.793 & $* * *$ \\
& & Families/Lines & 38 & 97.3 & 2.56 & 1.827 & $*$ \\
Trial $\times$ Families & 77 & Lines $\times$ Trials & 39 & 65.19 & 1.67 & 1.491 & NS \\
& & (Families/Lines) $\times$ Trials & 38 & 42.68 & 1.12 & 1.651 & $* *$ \\
Error & 2209 & Error & 2209 & 1498.75 & 0.678 & & \\
Total & 2364 & Total & 2364 & 2481.78 & & & \\
\hline
\end{tabular}

(b)

\begin{tabular}{lrccc}
\hline Item & d.f & MS & $F$ & $P$ \\
\hline Trial & 1 & 0.039 & 0.028 & NS \\
Family & 4 & 5.065 & 3.69 & NS \\
Trial $\times$ Family & 4 & 1.372 & 3.09 & $*$ \\
Error & 267 & 0.444 & & \\
Total & 276 & & & \\
\hline
\end{tabular}

respectively). ANOVAS were carried out using the combined data from the two trials. Lines SL115, SL121, SL127, SL133, SL134, SL138, SL141, SL142, SL158, SL172, SL175 and SL177 were shown to be significantly later flowering than the A12 parent, whereas SL108 and SL122 were significantly earlier flowering.

\section{Significant substitution lines}

Table 3 shows the mean of the A12 parent in each year, together with the means for each line that differed significantly in flowering time from the A12. Over the eight trials it can be seen that only two substitution lines (SL108 and SL122) were significantly earlier flowering, whereas 21 were significantly later flowering than the A12 parent (SL102, SL112, SL113, SL115, SL121, SL127, SL128, SL129, SL133, SL134, SL137, SL138, SL140, SL141, SL142, SL157, SL158, SL172, SL175, SL177, SL178).

\section{Discussion}

Seventy-nine lines were grown over the five years. Of these, only two lines showed evidence for earlier flowering than A12, whereas 21 lines were significantly later flowering. This bias was to be expected because sections of chromosomes from the late flowering GD parent had been substituted into the early flowering A12 recurrent parent.
Of the 23 lines that showed significant variation in flowering behaviour from the A12 parent, 10 have a single substitution, four have two substitutions, eight have three regions and one has four regions substituted with GD. The following interpretation should be read in conjunction with Fig. 1

\section{Lines with one substitution}

Any variation between the A12 and the 10 lines containing just one substitution must result from GD DNA in this region, therefore such lines enable the GD DNA region to be treated as a single segregating factor. Because it is not possible to define the proportion of GD DNA in the region in which recombination has occurred, it was assumed that any QTL present may be anywhere within the maximum substitution.

The late flowering line SL102 had a maximum GD substitution in the region between 0.0 and $35.7 \mathrm{cM}$ on chromosome O1, but the early flowering line SL108 had a minimum substitution between 30.3 and $35.7 \mathrm{cM}$, so it is unlikely that a late flowering QTL was present in this region (Fig. 1, O1). It is therefore most likely that there was a late flowering QTL between 0.0 and $30.3 \mathrm{cM}$ and an early flowering QTL between 30.3 and $38.1 \mathrm{cM}$ on chromosome O1.

Lines SL128 and SL133 both showed late flowering behaviour. Assuming the presence of just one QTL on

(C) The Genetical Society of Great Britain, Heredity, 83, 586-596. 
Table 3 The mean flowering date for the Brassica oleracea recurrent parent, A12, and the substitution lines that showed significantly different flowering behaviour from A12 over the five years. Bold type denotes those lines that were significant and '-' indicates that the line was not raised in that year

\begin{tabular}{lcccccc}
\hline Line & 1998 & 1997 & $1996 \mathrm{~T} 1$ & $1996 \mathrm{~T} 2$ & 1995 & 1994 \\
\hline A12 & 64.52 & 65.00 & 56.60 & 55.68 & 60.00 & 57.40 \\
SL102 & 65.07 & 65.19 & $\mathbf{6 0 . 3 2}$ & $\mathbf{5 9 . 0 9}$ & - & - \\
SL108 & $\mathbf{6 3 . 6 1}$ & 64.32 & 55.85 & 59.85 & $\mathbf{5 2 . 9 3}$ & 52.06 \\
SL112 & - & - & - & - & - & $\mathbf{7 4 . 6 9}$ \\
SL113 & - & - & - & - & $\mathbf{7 0 . 0 0}$ & $\mathbf{7 3 . 3 9}$ \\
SL115 & $\mathbf{6 6 . 5 3}$ & $\mathbf{6 8 . 8 8}$ & - & - & - & - \\
SL121 & $\mathbf{6 8 . 9 4}$ & 67.51 & - & - & - & - \\
SL122 & $\mathbf{6 3 . 3 7}$ & 65.17 & - & - & - & - \\
SL127 & $\mathbf{6 6 . 3 9}$ & - & - & - & - & - \\
SL128 & - & - & - & $\mathbf{5 8 . 5 9}$ & - & - \\
SL129 & - & - & - & - & $\mathbf{6 6 . 1 8}$ & 59.00 \\
SL133 & $\mathbf{6 6 . 0 0}$ & 64.35 & - & - & - & - \\
SL134 & $\mathbf{7 0 . 4 7}$ & $\mathbf{7 0 . 4 2}$ & - & - & - & - \\
SL137 & - & - & - & $\mathbf{5 9 . 3 8}$ & - & - \\
SL138 & $\mathbf{7 1 . 1 4}$ & 66.67 & - & - & - & - \\
SL140 & - & - & - & - & $\mathbf{6 6 . 9 3}$ & $\mathbf{6 9 . 6 9}$ \\
SL141 & $\mathbf{6 7 . 8 6}$ & $\mathbf{6 9 . 1 2}$ & - & - & - & - \\
SL142 & $\mathbf{6 7 . 7 6}$ & 66.53 & - & - & - & - \\
SL157 & - & - & - & $\mathbf{5 9 . 5 0}$ & - & - \\
SL158 & $\mathbf{6 8 . 1 3}$ & 64.79 & - & - & - & - \\
SL172 & $\mathbf{6 5 . 8 5}$ & 65.63 & 57.47 & $\mathbf{5 8 . 4 9}$ & $\mathbf{6 8 . 5 4}$ & 60.40 \\
SL175 & $\mathbf{6 7 . 4 0}$ & $\mathbf{6 8 . 1 6}$ & - & $\mathbf{6 1 . 2 9}$ & 57.30 & $\mathbf{6 8 . 7 5}$ \\
SL177 & $\mathbf{6 6 . 6 8}$ & 65.55 & - & - & 59.21 & - \\
SL178 & - & - & - & $\mathbf{5 9 . 5 0}$ & - & - \\
\hline
\end{tabular}

chromosome $\mathrm{O} 3$, it is likely to be located in the region in which the substitutions in these lines overlap, i.e. between 30.5 and $43.3 \mathrm{cM}$, although the presence of a second QTL in this region is discussed later (Fig. 1, O3).

Line SL157 suggested the presence of a late flowering QTL between 0.0 and $34.2 \mathrm{cM}$ on chromosome $\mathrm{O} 5$ in GD (Fig. 1. O5).

The five late flowering lines with single substitutions on chromosome $\mathrm{O} 9$ suggest three separate regions to which QTL may be mapped. Lines SL142, SL175 and SL178 have substitutions which overlap between 70.8 and $106.8 \mathrm{cM}$; line SL172 has a substitution between 0.0 and $43.0 \mathrm{cM}$; and line SL177 has a substitution between 43.0 and $64.4 \mathrm{cM}$ (Fig. 1, O9).

If these seven QTL are assumed to be present in the regions described, inferences can now be made about those lines which contain more than one QTL.

\section{Lines with more than one substitution}

Of the 13 significant lines with more than one GD substitution, the behaviour of 11 can be explained by
QTL found in lines with single substitutions. Inferences for QTL in the two lines SL122 and SL127 cannot be made using substitution line data alone, but are discussed below.

\section{Comparison to previous work}

Previous work carried out on this A12 $\times$ GD cross by Bohuon et al. (1998) analysed data from field trials using a mapping method based on marker regression (Kearsey $\&$ Hyne, 1994). This method showed evidence for six QTL over the two trials analysed in 1994. Single QTL were found on chromosomes $\mathrm{O} 2$ and $\mathrm{O} 3$, whereas chromosomes $\mathrm{O} 5$ and $\mathrm{O} 9$ both showed evidence for two QTL.

Figure 1 provides a comparison of the doubled haploid and substitution line data. The QTL are 'named' according to their chromosome and position in the two doubled haploid trials simply to facilitate comparisons (see Bohuon et al., 1998).

\section{Chromosome 01}

The doubled haploid data showed no evidence for QTL on chromosome O1, whereas the substitution line data showed the presence of two QTL; one late flowering between 0.0 and $30.3 \mathrm{cM}$ and one early flowering between 30.3 and $38.1 \mathrm{cM}$. As the regions to which these two putative QTLs map are adjacent to each other, they are likely to cancel each other in the doubled haploid lines.

\section{Chromosome $\mathrm{O} 2$}

The QTL FTO2.1, which had a late flowering allele from GD, was located at $78 \pm 9 \mathrm{cM}$ on chromosome O2. Eight of the substitution lines have introgressed GD DNA at this position. Of these, four flowered significantly late, one flowered early and three did not show significant variation from A12. The five that showed significant flowering variation all have substitutions on other chromosomes which may be affecting flowering time. SL112 and SL113 are thought to contain a region coding for late flowering on chromosome O3; SL121 contains late flowering regions on chromosomes $\mathrm{O} 1$ and $\mathrm{O} 9$; and the early flowering of SL122 may be caused by a QTL mapped in the region of FTO9.1 in the doubled haploid lines. It is possible that SL127 is reflecting the late flowering effect of FTO2.1. It may also be possible that lines SL112 and SL113 show this effect as well as the late flowering QTL on chromosome O3, because the mean flowering dates for lines SL112 and SL113 were 17 and 16 days later than A12, respectively, in 1994, whereas the other significantly late lines in this year flowered just 12-13 days later than A12. 

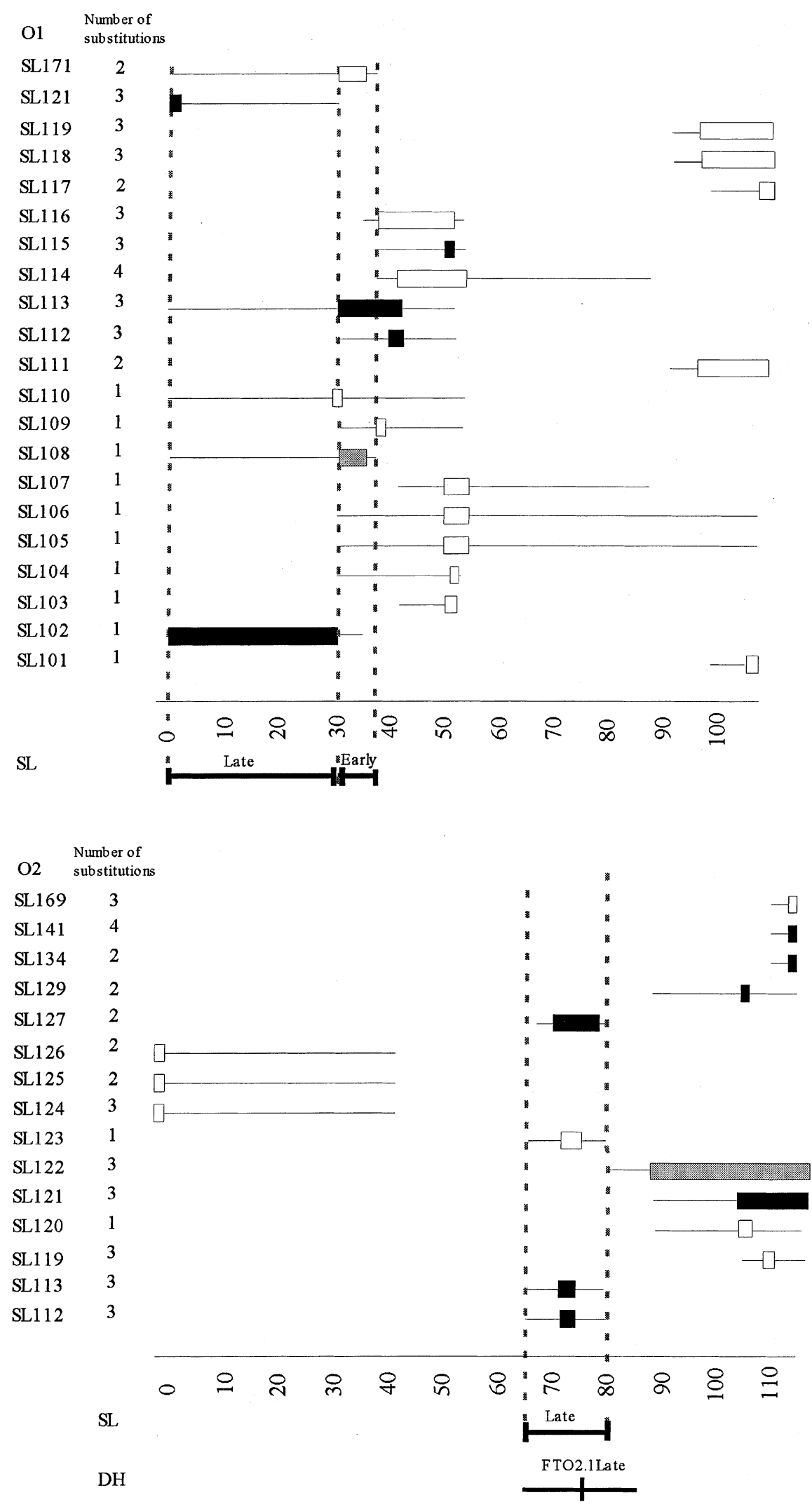

Fig. 1 (see caption on p. 593)

(c) The Genetical Society of Great Britain, Heredity, 83, 586-596. 


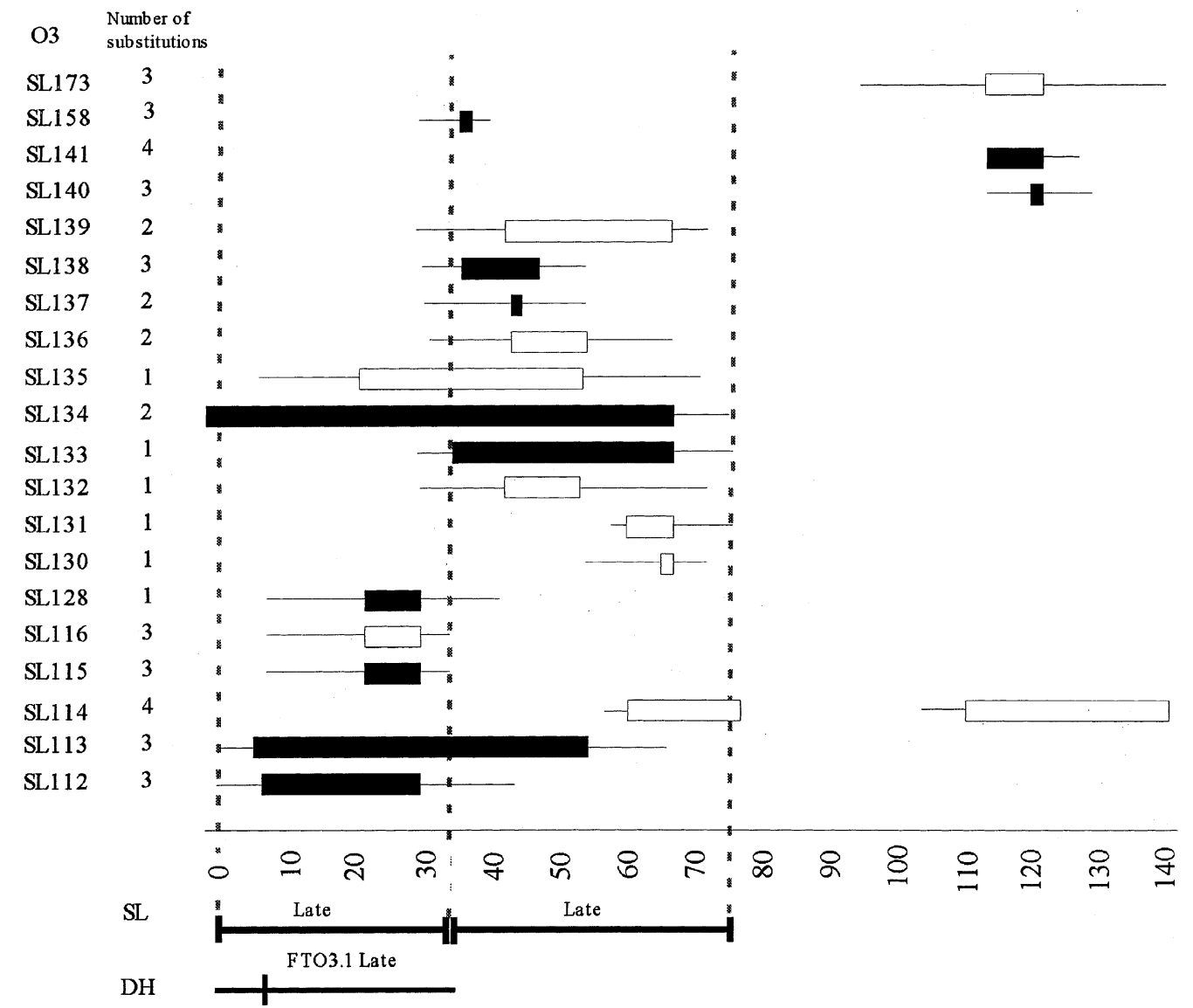

O5 lumber of

SL161

SL160 3

SL159 2

SL158 3

SL1 57

SL156 1

SL155 2

SL154

SL1 53

SL152

SL1 51

SL1 50

SL149

SL138

SL137

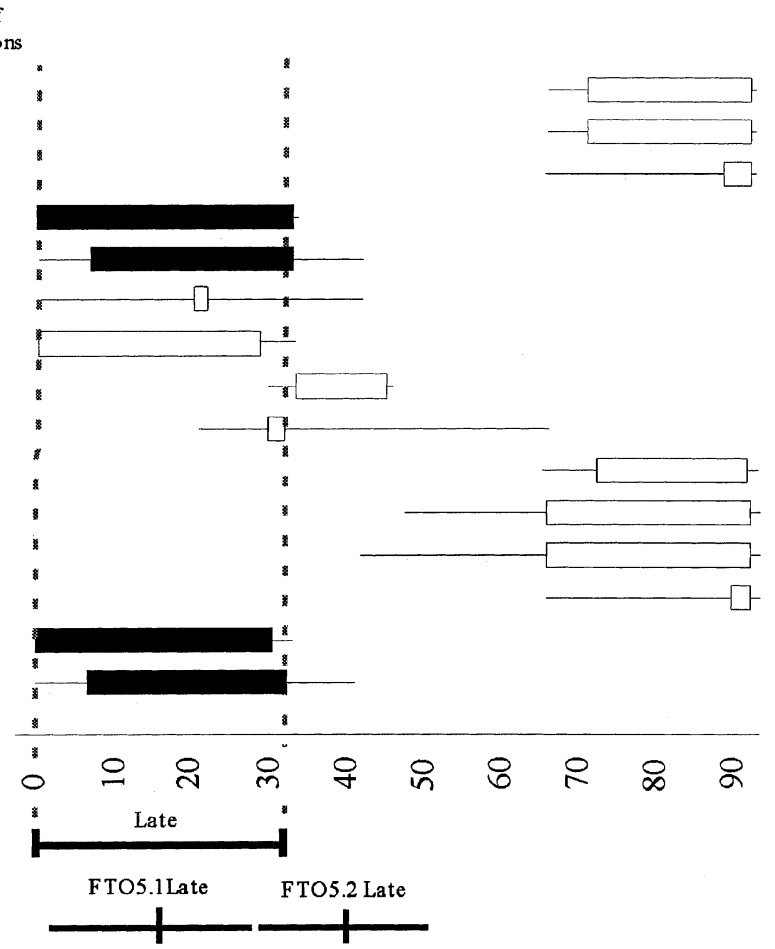

Fig. 1 (see caption on p. 593)

(C) The Genetical Society of Great Britain, Heredity, 83, 586-596. 


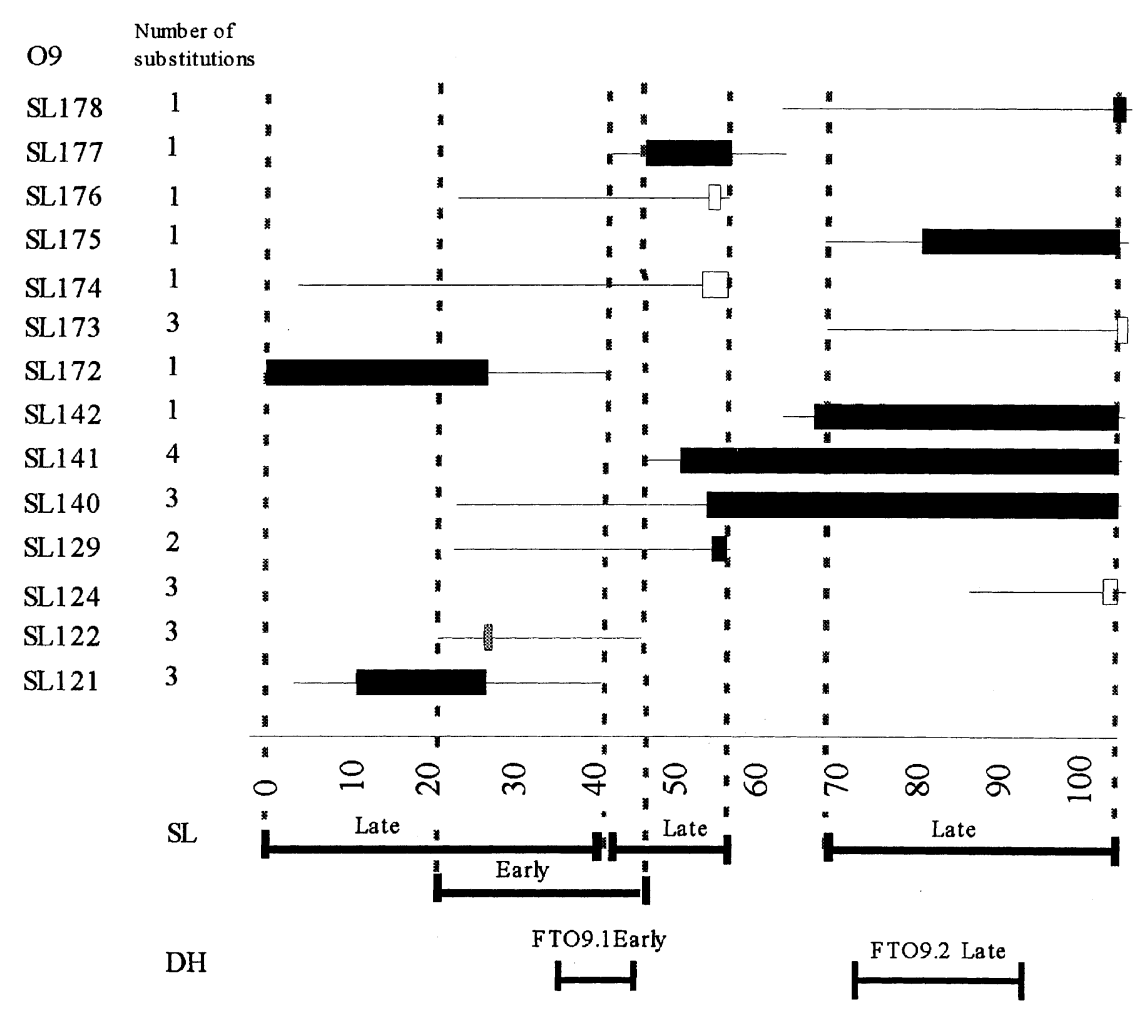

Fig. 1 The positions of introgressed regions in the Brassica oleracea substitution lines on chromosomes O1, O2, O3, O5 and O9. Minimum GD regions are shown as a box, whereas the maximum region, including where recombination occurred, is shown as a single line. The number of substitutions contained by each line is noted. The significantly late flowering lines are shaded in black, whereas those that were significantly early flowering are shaded in grey. The regions to which the substitution lines best map QTL are shown by vertical, dotted lines. For comparison, the regions to which the doubled haploid data mapped QTL are also shown.

\section{Chromosome $\mathrm{O} 3$}

The late flowering lines SL128 and SL133 have single substitutions which overlap, suggesting that FTO3.1 is in the overlapping region, but the maximum substitution in the late flowering line SL115 only just overlaps the maximum substitution in SL133. There is no evidence that the GD substitutions on chromosome $\mathrm{O} 1$ or $\mathrm{O} 6$ in SL115 affect the flowering behaviour of this line, therefore there may be two QTL affecting flowering time on this chromosome. This view is supported by the size of the effects of the QTL on this chromosome. The lines SL115 and SL133 flowered 2 and 1.5 days later than the A12 parent, respectively, in 1998, whereas line SL134, which overlaps both regions, flowered 6 days later than the A12 parent, implying the presence of two QTL in this line. The presence of a second QTL on chromosome O3 may explain the large confidence interval of FTO3.1 in the doubled haploid data.

\section{Chromosome 05}

FTO5.1 maps to $16 \pm 13 \mathrm{cM}$, whereas FTO5.2 maps to $40 \pm 10 \mathrm{cM}$. The substitution line data suggest that there is a QTL between 0.0 and $34.2 \mathrm{cM}$. This region overlaps with the confidence intervals for both FTO5.1 and FTO5.2, so the substitution lines neither confirm nor reject the two-QTL hypothesis.

\section{Chromosome 09}

The doubled haploid data indicated the presence of two QTL on chromosome O9; an early QTL (FTO9.1) at $46 \mathrm{cM}$ in trial 1 and $36 \mathrm{cM}$ in trial 2, and a late QTL (FTO9.2) at $74 \mathrm{cM}$ in trial 1 and $94 \mathrm{cM}$ in trial 2. The marker regression approach used on these doubled haploid data does not give confidence intervals when more than one QTL is mapped to a chromosome. The substitution-line data map a late flowering QTL between 70.8 and $106.8 \mathrm{cM}$, which coincides with the doubled haploid QTL FTO9.2.

The doubled haploid data did not reveal the two late QTL found in the substitution lines in the regions of $0.0-43.0 \mathrm{cM}$ and $43.0-64.4 \mathrm{cM}$, but did detect an early effect at $46 \mathrm{cM}$ in trial 1 and $36 \mathrm{cM}$ in trial 2. This may be the cause of early flowering in SL122, which has a GD substitution between 23.1 and $47.0 \mathrm{cM}$ on chromosome O9. The late flowering line SL129 completely

(C) The Genetical Society of Great Britain, Heredity, 83, 586-596. 
overlaps the region to which this early flowering QTL is mapped, but it is possible that this line contains the two late flowering QTLs at this end of chromosome O9, which mask the effect of early flowering.

There is some evidence that the doubled haploid lines show segregation distortion in favour of GD in the region of the early QTL, which may explain why the early QTL alone is detected in the doubled haploid data.

\section{Precision of mapping}

The doubled haploid data mapped QTL with 95\% confidence intervals that varied between 18 and $52 \mathrm{cM}$. Usually a confidence interval of $30 \mathrm{cM}$ is thought to be reasonable for analysis of segregating populations. In the substitution lines the interval to which a QTL can be mapped relies on the size of the substitution and also the distance between markers on the linkage map. Substitution lines that showed significant effects for flowering time contained introgressed GD DNA that ranged in length from $8 \mathrm{cM}$ to $43 \mathrm{cM}$, showing that this method of mapping QTL does enable effects to be mapped more precisely. The substitution line approach identified at least 11 QTL compared to the six detected by the doubled haploids. Substitution lines identified more QTL, because they detected effects that had been masked in the doubled haploid population through dispersion and close linkage.

It is possible that there are still more QTL for flowering time segregating in this cross. Some of the substitution lines contained introgressed regions as long as $84 \mathrm{cM}$, so it is quite feasible that such large regions may contain more than one QTL, which mask each other's effect. The substitution lines grown over the five years represent $62 \%$ of the entire GD genome, although the actual proportion may be larger because this calculation does not include the region within which recombination has occurred. Only $9.0 \%$ of the GD genome is definitely not present in the substitution lines. This implies that there may well be QTL in the regions that are not represented in the substitution lines.

\section{Comparison to other genomes}

The use of the linkage map produced by Bohuon et al. (1996) in this study has enabled a comparison of results to be made. This map has also been used to compare the $\mathrm{C}$ genomes of B. oleracea and B. napus, and colinearity of the large segments of the $\mathrm{A}$ and $\mathrm{C}$ genomes in B. napus (Parkin et al., 1995). Field experiments involving Brassica napus populations have indicated that regions on linkage groups in the A genome which show homology to the regions in $\mathrm{O} 2, \mathrm{O} 3$ and $\mathrm{O} 9$ also carry QTL for flowering time (Keith, 1996; Salinas-Garcia,
1996; Osborn et al., 1997). These regions have also been shown to be homologous with regions on LG2 and LG8 of B. nigra, to which a flowering time QTL has been mapped (Lagercrantz et al., 1996) and to the region around the CONSTANS gene and several other flowering candidate genes on chromosome 5 in $A$. thaliana. These regions of homology have been found to map to the regions of B. oleracea chromosomes $\mathrm{O} 2, \mathrm{O} 3$ and $\mathrm{O} 9$, and to show similar effects of late flowering in GD (Bohuon et al., 1998). These appear to be the same as the substituted regions in the present study that also showed evidence of QTL for late flowering in GD.

The substitution line data showed similar results to the doubled haploid data, but were not as easily interpreted. To maximize the benefits of the use of substitution lines, it would be advantageous to backcross lines further to reduce the size and number of introgressed GD regions in each line, and to map more markers to the linkage map to enable reduction of the regions in which recombination is presumed to have occurred.

\section{Other measurements}

Measurements for height and number of nodes at flowering were also taken in the eight substitution line trials, as these have been found to have a positive correlation with flowering time (Bohuon, 1996). Late flowering tends to be a result of extended vegetative growth rather than stunted or delayed growth (Kowalski et al., 1994). Future work will involve the analysis of these traits and a comparison to flowering time data.

\section{Acknowledgements}

The authors particularly thank Judith Craft and Sue Bradshaw for technical support. This work was supported by the UK Biotechnology and Biological Sciences Council (BBSRC) research grant number 6/G02511.

\section{References}

BOHUON, E. J. R. 1996. A Genetic Analysis of Brassica oleracea. Ph.D. Thesis, University of Birmingham.

BOHUON, E. J. R., KEITH, D. J., PARKIN, I. A. P., SHARPE, A. G. AND LYDiATE, D. J. 1996. Alignment of the conserved C genomes of Brassica oleracea and Brassica napus. Theor. Appl. Genet., 93, 833-839.

BOHUON, E. J. R., RAMSAY, L. D., CRAFT, J. A., ARTHUR, A. E., MARSHALL, D. F., LYDIATE, D. J. ET AL. 1998. The association of flowering time quantitative trait loci with duplicated regions and candidate loci in Brassica oleracea. Genetics, 150, 393-401.

CAMARGo, L. E. A. AND OSBorn, T. C. 1996. Mapping loci controlling flowering time in Brassica oleracea. Theor. Appl. Genet., 92, 610-616.

(c) The Genetical Society of Great Britain, Heredity, 83, 586-596. 
DUNNETT, C. W. 1980. Pairwise multiple comparisons in the homogeneous variance, unequal sample size case. J. Am. Stat. Ass., 75, 789-795.

KEARSEY, M. J. AND HYNE, v. 1994. QTL analysis: a simple 'marker-regression' approach. Theor. Appl. Genet., 89, 698-702.

KEARSEY, M. J. AND POONI, H. S. 1996. The Genetical Analysis of Quantitative Traits. Chapman \& Hall, London.

KEITH, D. J. 1996. Genetical Analysis of Quantitative Traits in Brassica napus. Ph.D. Thesis, University of East Anglia.

KENNARD, W. C., SLOCUM, M. K., FIGDORE, S. S. AND OSBORN, T. C. 1994. Genetic analysis of morphological variation in Brassica oleracea using molecular markers. Theor. Appl. Genet., 87, 721-732.

KOORNNEEF, M., HANHART, C. J. AND VAN DER VEEN, J. H. 1991. A genetic and physiological analysis of late flowering mutants in Arabidopsis thaliana. Mol. Gen. Genet., 229, 57-66.

KOWALSKI, S. P., LAN, T. H., FELDMANN, K. A. AND PATERSON, A. H. 1994. QTL mapping of naturally-occurring variation in flowering time in Arabidopsis thaliana. Mol. Gen. Genet., 245, 548-555.

LAGERCRANTZ, U. AND LYDIATE, D. J. 1996. Comparative genome mapping in Brassica. Genetics, 144, 1903-1910.

LAGERCRANTZ, U., PUTTERILl, J., COUPLAND, G. AND LYDiATE, D. 1996. Comparative mapping in Arabidopsis and Brassica, fine scale colinearity and congruence of genes controlling flowering time. Plant J., 9, 13-20.

MURPHY, L. A. AND SCARTH, R. 1994. Vernalisation response in spring oilseed rape (Brassica napus L.) cultivars. Can. J. Plant Sci., 74, 275-277.

OSBORN, T. C., KOLE, C., PARKIN, I. A. P., SHARPE, A. G., KUIPER, M., LYDiATE, D. J. ET $A L$. 1997. Comparison of flowering time genes in Brassica rapa. B. napus and Arabidopsis thaliana. Genetics, 146, 1123-1129.
PARKIN, I., SHARPE, A. G., KEITH, D. J. AND LYDIATE, D. J. 1995. Identification of the $\mathrm{A}$ and $\mathrm{C}$ genomes of amphidiploid Brassica napus (oilseed rape). Genome, 38, 1122-1131.

PUTTERILl, J., ROBSON, F., LEE, K. AND COUPLAND, G. 1993. Chromosome walking with YAC clones in Arabidopsis: isolation of $1700 \mathrm{~kb}$ of contiguous DNA on chromosome 5, including a $300 \mathrm{~kb}$ region containing the flowering time gene CO. Mol. Gen. Genet., 239, 145-157.

PUTTERILL, J., ROBSON, F., LEE, K., SIMON, R. AND COUPLAND, G. 1995. The CONSTANS gene of Arabidopsis promotes flowering and encodes a protein showing similarities to zinc finger transcription factors. Cell, 80, 847-857.

RAMSAY, L. D., JENNINGS, D. E., BOHUON, E. J. R., ARTHUR, A. E., LYDIATE, D. J., KEARSEY, M. J. ET AL. 1996. The construction of a substitution library of recombinant backcross lines in Brassica oleracea for the precision mapping of quantitative trait loci. Genome, 39, 558-567.

SAlinAS-GARCIA, G. 1996. Mapping Quantitative Trait Loci Controlling Traits in Brassica napus L. Ph.D. Thesis, University of Birmingham.

SEBASTIAN, R. L., HOWELL, E. C., KING, G. J., MARSHALL, D. F. AND KEARSEY, M. J. 1999. An integrated AFLP and RFLP Brassica oleracea linkage map from two morphologically distinct doubled haploid populations. Theor. Appl. Genet., in press.

THURLING, N. AND DEPITTAYANAN, v. 1992. EMS induction of early flowering mutants in spring rape (Brassica napus). Pl. Breed., 108, 177-184.

U, N. 1935. Genome analysis in Brassica with special reference to the experimental formation of B. napus and peculiar mode of fertilisation. Jap. J. Bot., 7, 389-452.

\section{Appendix 1}

\begin{tabular}{lcccccc}
\hline Line & Chromosome & $\begin{array}{c}\text { Minimum } \\
\text { substitution }\end{array}$ & $\begin{array}{c}\text { Maximum } \\
\text { substitution }\end{array}$ & Line & Chromosome & $\begin{array}{c}\text { Minimum } \\
\text { substitution }\end{array}$ \\
\hline SL100 & - & - & - & SL138 & O3 & $35.9-47.3$ \\
substitution
\end{tabular}

(C) The Genetical Society of Great Britain, Heredity, 83, 586-596. 
Appendix 1 (Continued)

\begin{tabular}{|c|c|c|c|c|c|c|c|}
\hline Line & Chromosome & $\begin{array}{l}\text { Minimum } \\
\text { substitution }\end{array}$ & $\begin{array}{l}\text { Maximum } \\
\text { substitution }\end{array}$ & Line & Chromosome & $\begin{array}{l}\text { Minimum } \\
\text { substitution }\end{array}$ & $\begin{array}{l}\text { Maximum } \\
\text { substitution }\end{array}$ \\
\hline SL113 & $\mathrm{O} 3$ & $7.5-54.2$ & $0.0-67.2$ & SL148 & O4 & 100.1 & $44.1-100.1$ \\
\hline SL114 & $\mathrm{O} 3$ & $60.2-78.9$ & $58.2-78.9$ & SL150 & O5 & $67.5-91.2$ & $42.7-92.9$ \\
\hline SL114 & $\mathrm{O} 3$ & $113.2-140.1$ & $104.3-140.1$ & SL151 & $\mathrm{O} 4$ & 44.1 & $39.5-100.1$ \\
\hline SL114 & O6 & $0.0-9.8$ & $0.0-14.8$ & SL151 & O5 & $67.5-91.8$ & $49.2-92.9$ \\
\hline SL115 & $\mathrm{O} 3$ & $22.5-30.5$ & $7.5-35.9$ & SL152 & O5 & $73.5-91.8$ & $67.5-92.9$ \\
\hline SL115 & O6 & 18.0 & $14.8-20.2$ & SL153 & O5 & 33.6 & $21.0-67.5$ \\
\hline SL116 & $\mathrm{O} 1$ & $38.1-50.6$ & $35.7-52.0$ & SL154 & O5 & $34.2-43.6$ & $30.9-44.7$ \\
\hline SL116 & $\mathrm{O} 3$ & $22.5-30.5$ & $7.5-35.9$ & SL155 & O5 & $0.0-30.9$ & $0.0-34.2$ \\
\hline SL116 & O6 & 18.0 & $14.8-20.2$ & SL155 & O6 & 18.0 & $14.8-20.2$ \\
\hline SL117 & $\mathrm{O} 1$ & $107.7-108.6$ & $99.3-108.6$ & SL156 & O5 & 21.0 & $0.0-42.7$ \\
\hline SL119 & $\mathrm{O} 1$ & $95.8-108.6$ & $90.7-108.6$ & SL159 & O5 & $90.7-91.2$ & $67.5-92.9$ \\
\hline SL119 & $\mathrm{O} 2$ & 110.4 & $105.2-116.2$ & SL159 & O8 & $1.9-19.2$ & $0.0-79.3$ \\
\hline SL119 & $\mathrm{O} 7$ & 5.9-8.4 & $0.0-15.7$ & SL160 & $\mathrm{O} 4$ & 44.1 & $39.5-100.1$ \\
\hline SL120 & $\mathrm{O} 2$ & 105.2 & $88.4-116.2$ & SL160 & O5 & $73.5-91.8$ & $67.5-92.9$ \\
\hline SL121 & $\mathrm{O} 1$ & 0.0 & $0.0-30.3$ & SL160 & O8 & $1.9-11.8$ & $0.0-17.7$ \\
\hline SL121 & $\mathrm{O} 2$ & $105.2-116.2$ & $88.4-116.2$ & SL161 & $\mathrm{O} 4$ & 44.1 & $39.5-100.1$ \\
\hline SL121 & O9 & 13.8-28.1 & $4.1-43.0$ & SL161 & O5 & $73.5-91.8$ & $67.5-92.9$ \\
\hline SL122 & $\mathrm{O} 2$ & $88.4-116.2$ & $80.8-116.2$ & SL161 & O8 & 1.9 & $0.0-4.3$ \\
\hline SL122 & O6 & $2.1-14.8$ & $0.0-18.0$ & SL162 & O6 & $2.1-32.8$ & $2.1-41.1$ \\
\hline SL122 & O9 & 28.1 & $23.1-47.0$ & SL162 & O8 & $1.9-11.8$ & $0.0-17.7$ \\
\hline SL123 & $\mathrm{O} 2$ & $73.0-74.0$ & $66.9-80.8$ & SL163 & $\mathrm{O} 7$ & 72.0 & $54.4-72.0$ \\
\hline SL127 & $\mathrm{O} 2$ & $70.2-79.4$ & $68.4-80.8$ & SL169 & $\mathrm{O} 2$ & 116.2 & $110.4-116.2$ \\
\hline SL127 & $\mathrm{O} 4$ & $39.5-44.1$ & $37.6-100.1$ & SL169 & O6 & 41.1 & $32.8-72.5$ \\
\hline SL128 & $\mathrm{O} 3$ & $22.5-30.5$ & $7.5-43.3$ & SL169 & $\mathrm{O} 7$ & $5.9-15.7$ & $0.0-36.5$ \\
\hline SL129 & $\mathrm{O} 2$ & 105.2 & $88.4-116.2$ & SL170 & $\mathrm{O} 7$ & $49.5-72.0$ & $38.2-72.0$ \\
\hline SL129 & O9 & 55.5 & $23.1-58.1$ & SL171 & $\mathrm{O} 1$ & $30.3-35.7$ & $0.0-38.1$ \\
\hline SL130 & $\mathrm{O} 3$ & 67.2 & $54.2-72.1$ & SL171 & O8 & 11.8 & $5.5-17.7$ \\
\hline SL131 & $\mathrm{O} 3$ & $60.2-67.2$ & $58.2-76.1$ & SL172 & O9 & $0.0-28.1$ & $0.0-43.0$ \\
\hline SL132 & $\mathrm{O} 3$ & $43.3-54.2$ & $30.5-72.1$ & SL173 & $\mathrm{O} 3$ & $114.7-121.2$ & $95.8-140.1$ \\
\hline SL133 & $\mathrm{O} 3$ & $35.9-67.2$ & $30.51-76.1$ & SL173 & O8 & $73.2-79.3$ & $35.5-79.5$ \\
\hline SL134 & $\mathrm{O} 2$ & 116.2 & $110.4-116.2$ & SL173 & O9 & 106.8 & $70.8-106.8$ \\
\hline SL134 & $\mathrm{O} 3$ & $0.0-67.2$ & $0.0-76.1$ & SL174 & O9 & 55.5 & $4.1-58.1$ \\
\hline SL135 & $\mathrm{O} 3$ & $22.5-54.2$ & $7.5-72.1$ & SL175 & O9 & $80.9-105.9$ & $70.8-106.8$ \\
\hline SL136 & $\mathrm{O} 3$ & $43.3-54.2$ & $30.5-67.2$ & SL176 & O9 & 55.5 & $23.1-58.1$ \\
\hline SL136 & $\mathrm{O} 4$ & 100.1 & $74.6-100.1$ & SL177 & O9 & $47.0-58.1$ & $43.0-64.4$ \\
\hline SL137 & $\mathrm{O} 3$ & 43.3 & $30.5-54.2$ & SL178 & O9 & 105.9 & $64.4-106.8$ \\
\hline SL137 & O5 & $7.0-33.6$ & $0.0-42.7$ & SL179 & O6 & $0.0-9.8$ & $0.0-14.8$ \\
\hline
\end{tabular}

(c) The Genetical Society of Great Britain, Heredity, 83, 586-596. 